\title{
Probing binding of Ethylated pillar[5]arene with Pentene and Chlorobutane Positional Isomers
}

\author{
Sulakshana V. Athare and Shridhar P. Gejji* \\ Department of Chemistry, Savitribai Phule Pune University, Pune, 411007.
}

\section{Supporting Information}

Figure S1: Frontier orbitals( $\pm 0.02 \mathrm{au})$ of complexes of pentene isomers with EtP5

Figure S2: Frontier orbitals $( \pm 0.02 \mathrm{au})$ of complexes of chlorobutane isomers with EtP5

Figure S3: Topological graphic of critical points for the complexes of EtP5 with isomers

Tabe S4: QTAIM parameters $\rho_{\mathrm{BCP}}, \nabla^{2} \rho_{\mathrm{BCP}}$, Ellipticity $(\epsilon), \mathrm{V}_{\mathrm{BCP}}, \mathrm{G}_{\mathrm{BCP}}, \mathrm{H}_{\mathrm{BCP}}$

Table S5: Summary of interactions from QTAIM analysis.

Figure S6: Color-filled RDG isosurface plot and NCI plots

Figure S7: IR spectra for pentene and chlorobutane complexes of EtP5 
HOMO

1-P@EtP5

t2p@EtP5

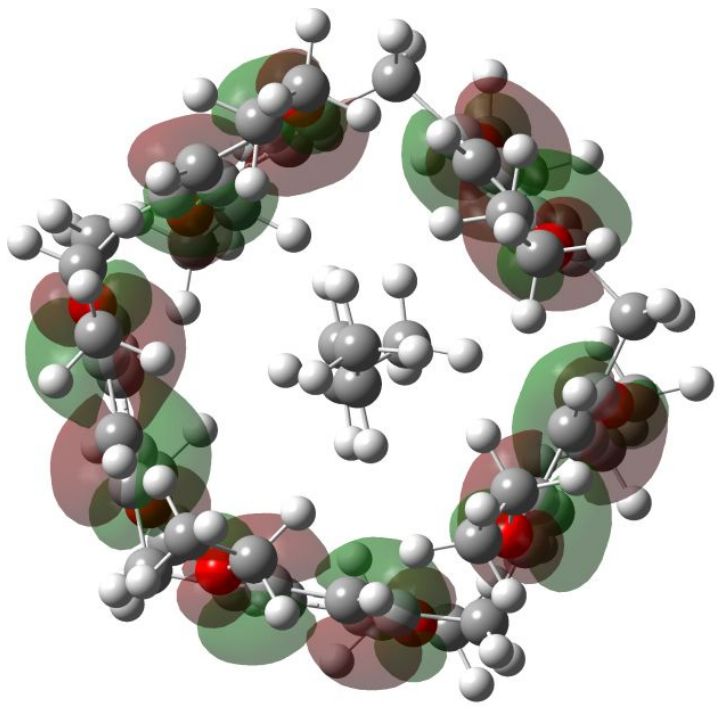

c2p@EtP5

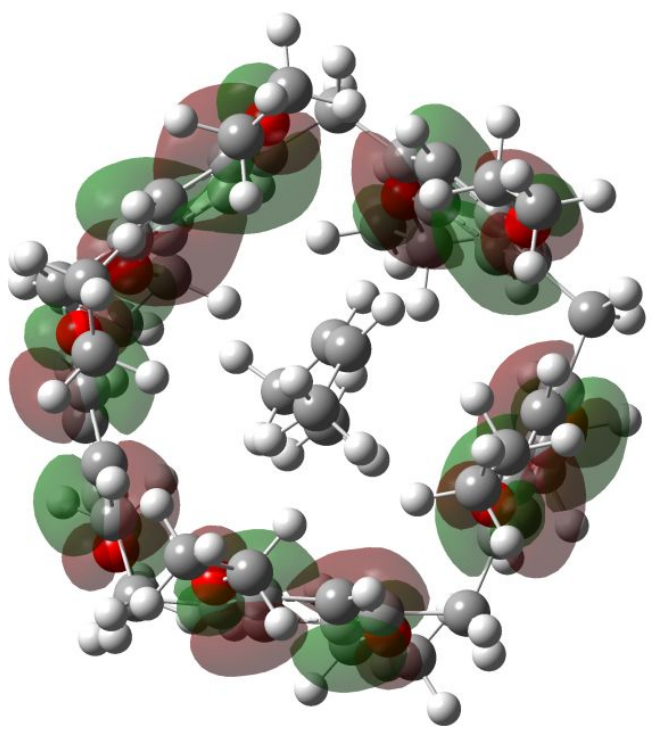

LUMO
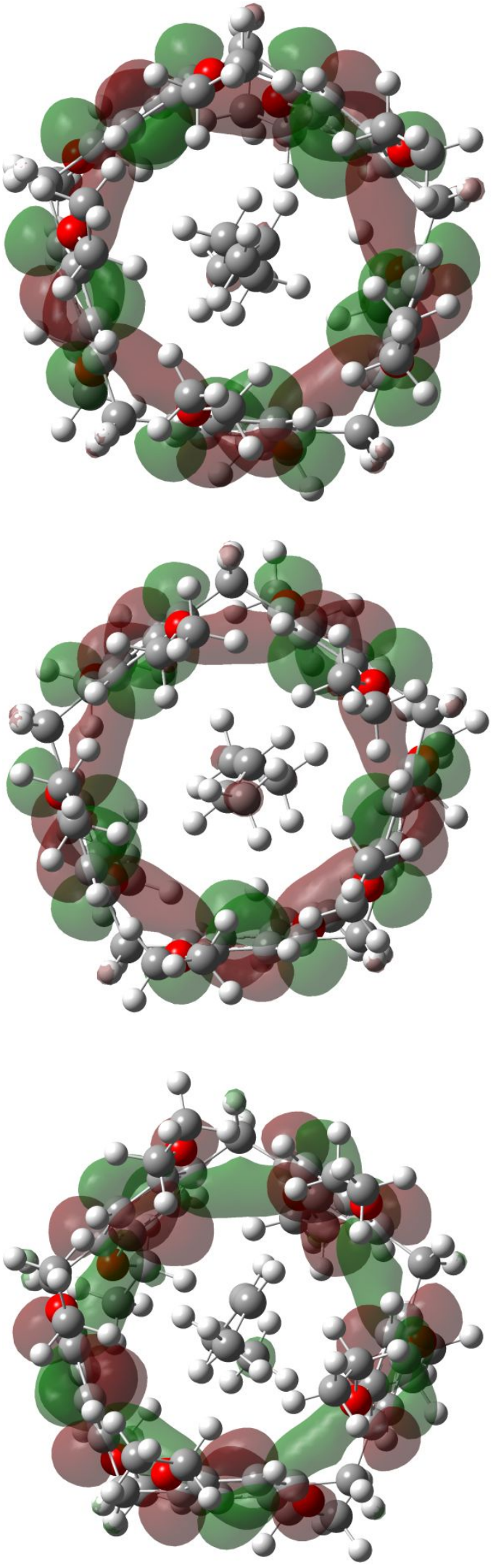

Fig. S1 Frontier orbitals $( \pm 0.02 \mathrm{au})$ of complexes of pentene isomers with EtP5 
HOMO

1CIBu@EtP5

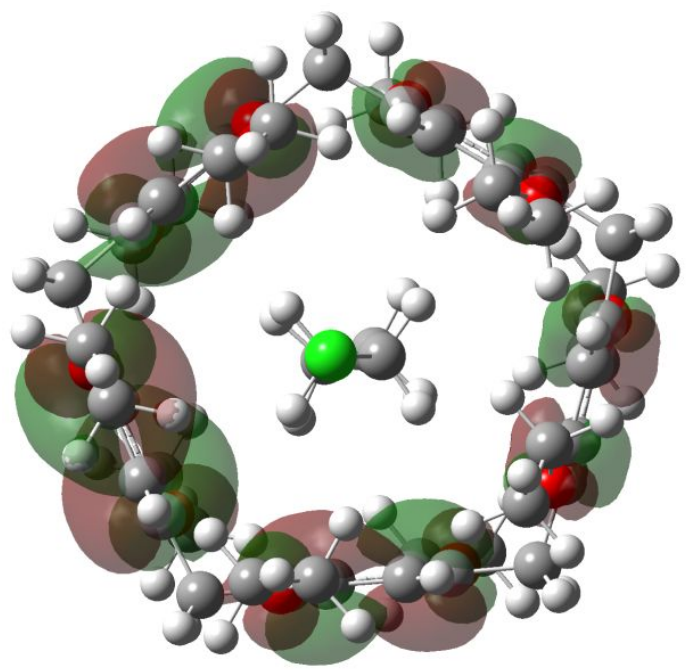

2ClBu@EtP5

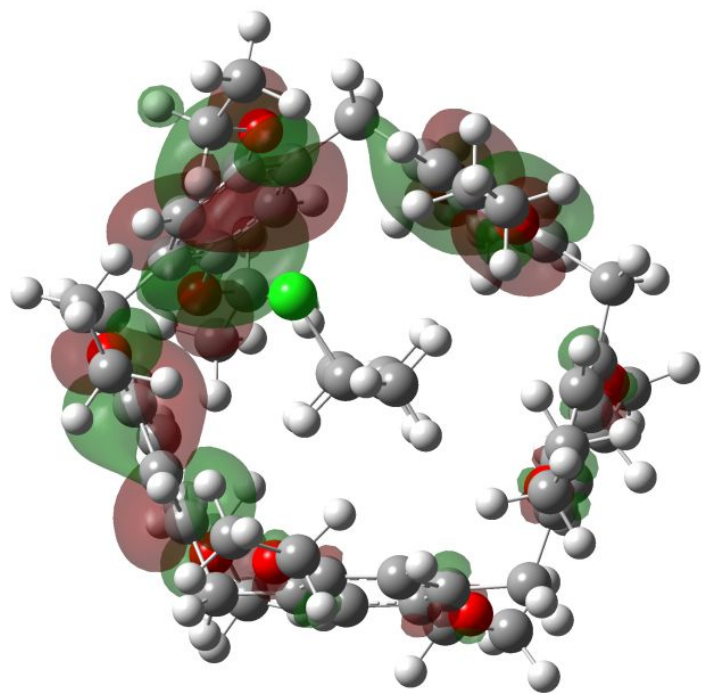

LUMO
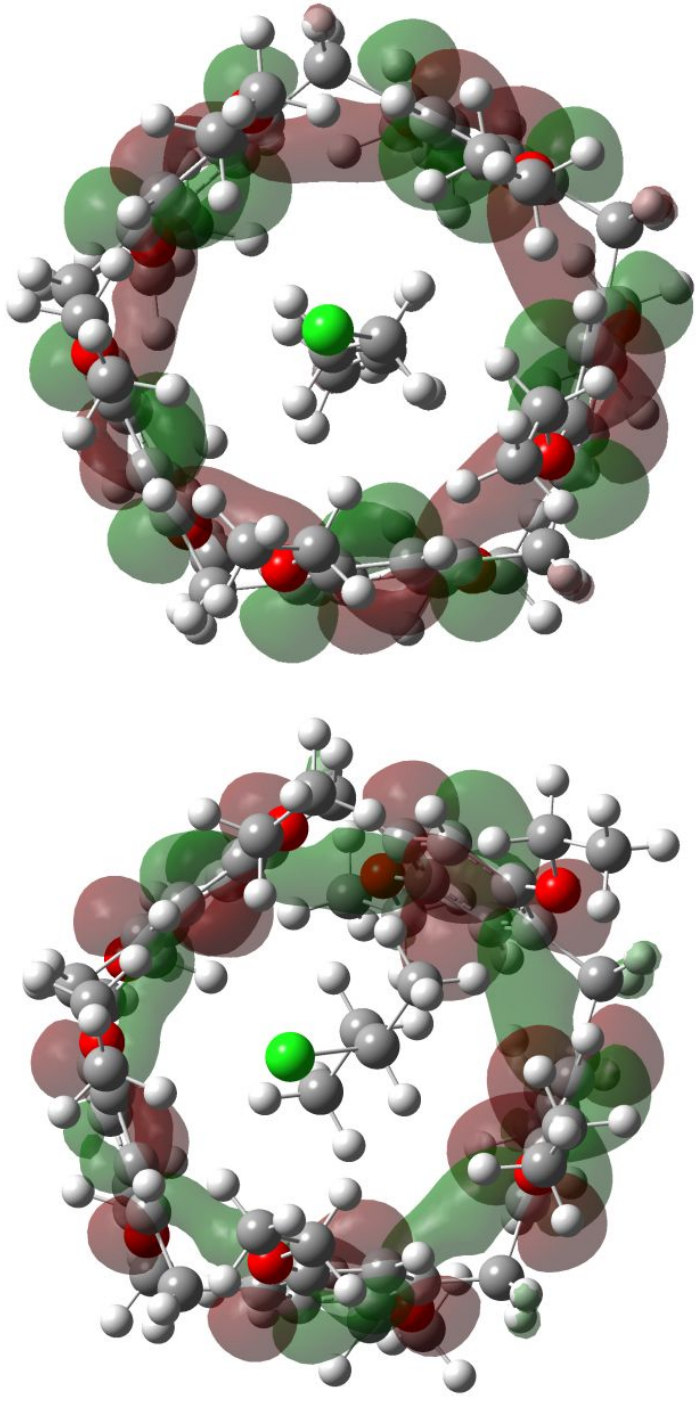

Fig. S2. Frontier orbitals $( \pm 0.02 \mathrm{au})$ of complexes of chlorobutane isomers with EtP5 


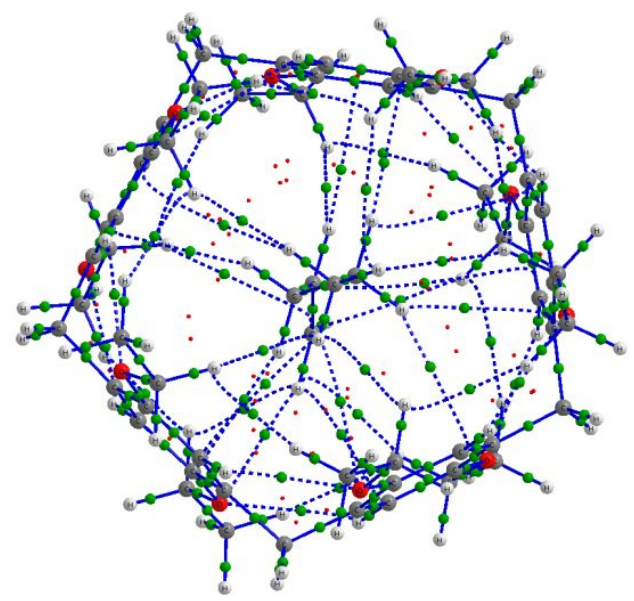

1-P@EtP5

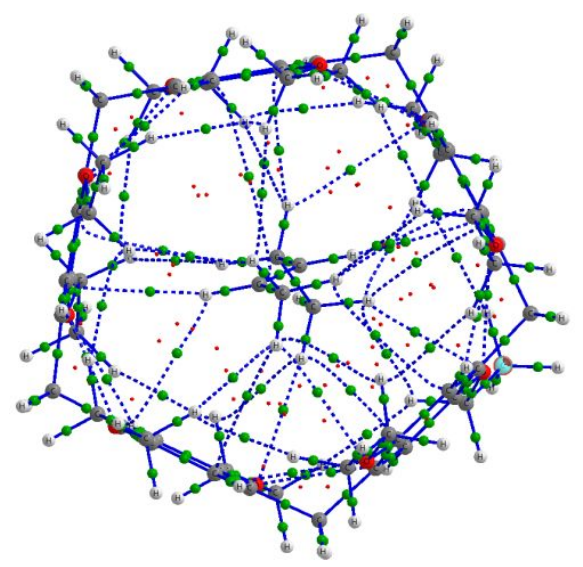

t2p@EtP5

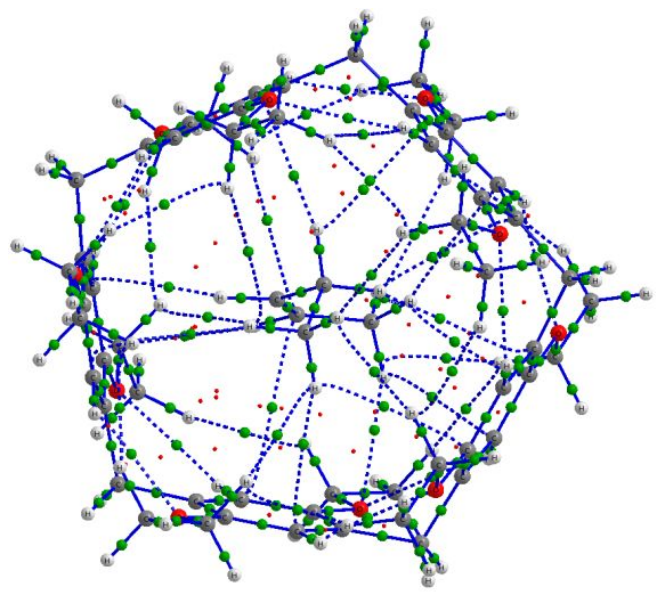

c2p@EtP5

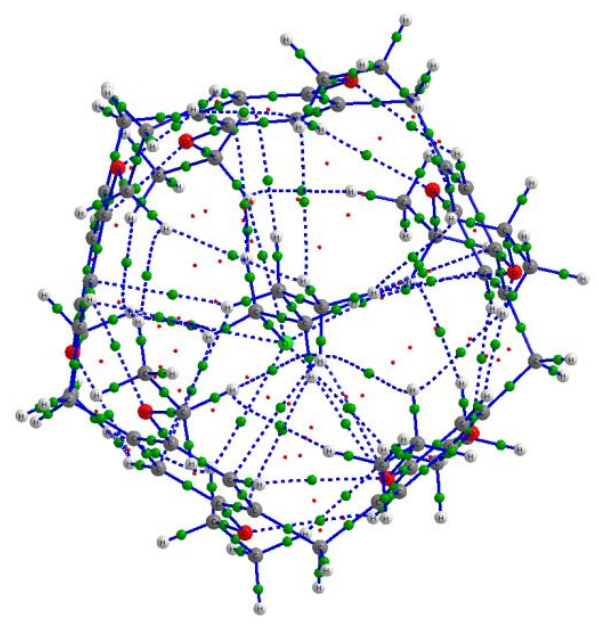

1CIBu@EtP5

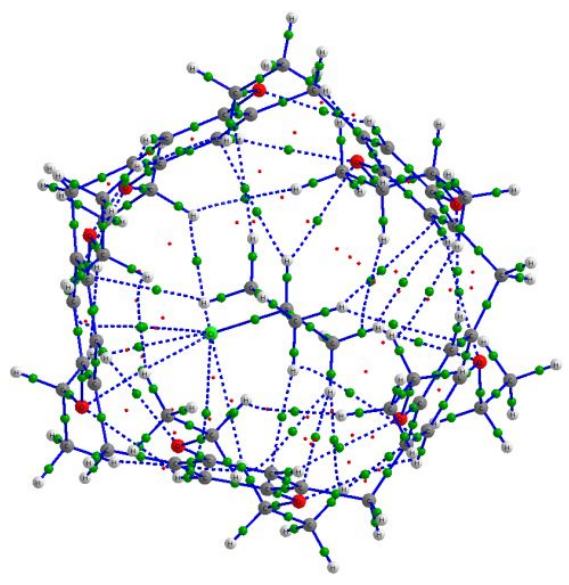

2ClBu@EtP5

Fig. S3 Topological graphic of critical points for the complexes of EtP5 with isomers 
Table S4. QTAIM parameters $\rho_{\mathrm{BCP}}, \nabla^{2} \rho_{\mathrm{BCP}}$, Ellipticity $(\epsilon), \mathrm{V}_{\mathrm{BCP}}, \mathrm{G}_{\mathrm{BCP}}, \mathrm{H}_{\mathrm{BCP}}$ are given in au.

\begin{tabular}{|c|c|c|c|c|c|c|c|c|c|}
\hline Interactions & $\rho_{\mathrm{BCP}}$ & $\nabla^{2} \rho_{\mathrm{BCP}}$ & $\epsilon$ & $\mathrm{V}_{\mathrm{BCP}}$ & $\mathrm{G}_{\mathrm{BCP}}$ & $\mathrm{H}_{\mathrm{BCP}}$ & $\left(-\mathrm{G}_{\mathrm{BCP}}\right) / \mathrm{V}_{\mathrm{BCP}}$ & $\mathrm{R}(\AA)$ & $\begin{array}{l}\mathrm{E}_{\mathrm{HB}}(\mathrm{kcal} \\
\left.\mathrm{mol}^{-1}\right)\end{array}$ \\
\hline \multicolumn{10}{|c|}{ 1-P@EtP5 } \\
\hline C52 - H142 & 0.0069 & 0.0185 & 1.2183 & -0.0033 & 0.0040 & 0.0007 & 0.8320 & 2.856 & 1.06 \\
\hline C4 - H142 & 0.0045 & 0.0126 & 1.7721 & -0.0022 & 0.0027 & 0.0005 & 0.8130 & 2.964 & 0.72 \\
\hline C10 - H141 & 0.0060 & 0.0163 & 2.3273 & -0.0029 & 0.0035 & 0.0006 & 0.8371 & 2.985 & 0.94 \\
\hline C17 - H141 & 0.0049 & 0.0126 & 1.5432 & -0.0023 & 0.0027 & 0.0004 & 0.8545 & 2.893 & 0.74 \\
\hline C27 - H145 & 0.0068 & 0.0188 & 1.8601 & -0.0034 & 0.0040 & 0.0007 & 0.8339 & 2.861 & 1.09 \\
\hline C28 - H138 & 0.0034 & 0.0087 & 0.0673 & -0.0015 & 0.0018 & 0.0003 & 0.8148 & 3.084 & 0.49 \\
\hline C38 - H144 & 0.0063 & 0.0188 & 4.3524 & -0.0033 & 0.0040 & 0.0007 & 0.8239 & 2.921 & 1.07 \\
\hline C18 - H149 & 0.0035 & 0.0100 & 0.1557 & -0.0016 & 0.0020 & 0.0005 & 0.7674 & 3.332 & 0.55 \\
\hline C41 - H139 & 0.0027 & 0.0072 & 0.1255 & -0.0012 & 0.0015 & 0.0003 & 0.7952 & 3.264 & 0.40 \\
\hline C51 - H147 & 0.0056 & 0.0142 & 0.9073 & -0.0025 & 0.0030 & 0.0005 & 0.8337 & 2.886 & 0.82 \\
\hline C7 - C146 & 0.0018 & 0.0044 & 0.9574 & -0.0007 & 0.0009 & 0.0002 & 0.7697 & 4.103 & 0.24 \\
\hline H82 - C148 & 0.0038 & 0.0081 & 0.3911 & -0.0016 & 0.0018 & 0.0002 & 0.8669 & 3.274 & 0.48 \\
\hline O63 - H149 & 0.0064 & 0.0200 & 0.5560 & -0.0041 & 0.0045 & 0.0005 & 0.8970 & 2.676 & 1.22 \\
\hline O60 - H138 & 0.0039 & 0.0138 & 0.7022 & -0.0025 & 0.0030 & 0.0005 & 0.8443 & 3.150 & 0.80 \\
\hline H70 - H149 & 0.0062 & 0.0180 & 0.4636 & -0.0031 & 0.0038 & 0.0007 & 0.8088 & 2.525 & 1.02 \\
\hline H92 - H137 & 0.0044 & 0.0111 & 0.0095 & -0.0022 & 0.0025 & 0.0003 & 0.8782 & 2.492 & 0.66 \\
\hline H68 - H138 & 0.0049 & 0.0146 & 0.4851 & -0.0026 & 0.0031 & 0.0006 & 0.8221 & 2.702 & 0.83 \\
\hline H89 - H147 & 0.0052 & 0.0140 & 0.1444 & -0.0027 & 0.0031 & 0.0004 & 0.8735 & 2.436 & 0.84 \\
\hline H95 - H150 & 0.0040 & 0.0108 & 0.9748 & -0.0018 & 0.0023 & 0.0004 & 0.8032 & 2.539 & 0.61 \\
\hline H76 - H149 & 0.0071 & 0.0223 & 0.5056 & -0.0039 & 0.0047 & 0.0009 & 0.8187 & 2.271 & 1.27 \\
\hline H86 - H137 & 0.0025 & 0.0079 & 0.7779 & -0.0012 & 0.0016 & 0.0004 & 0.7442 & 2.945 & 0.42 \\
\hline H73 - H138 & 0.0038 & 0.0106 & 0.1857 & -0.0019 & 0.0023 & 0.0004 & 0.8296 & 2.508 & 0.61 \\
\hline H79 - H139 & 0.0046 & 0.0119 & 0.0219 & -0.0023 & 0.0026 & 0.0003 & 0.8737 & 2.448 & 0.71 \\
\hline H89 - H147 & 0.0052 & 0.0140 & 0.1444 & -0.0027 & 0.0031 & 0.0004 & 0.8735 & 2.436 & 0.84 \\
\hline
\end{tabular}




\begin{tabular}{|c|c|c|c|c|c|c|c|c|c|}
\hline Interactions & $\rho_{\mathrm{BCP}}$ & $\nabla^{2} \rho_{\mathrm{BCP}}$ & $\epsilon$ & $\mathrm{V}_{\mathrm{BCP}}$ & $\mathrm{G}_{\mathrm{BCP}}$ & $\mathrm{H}_{\mathrm{BCP}}$ & $\left(-G_{B C P}\right) / V_{B C P}$ & $\mathrm{R}(\AA)$ & $\begin{array}{l}\mathrm{E}_{\mathrm{HB}}(\mathrm{kcal} \\
\left.\mathrm{mol}^{-1}\right)\end{array}$ \\
\hline \multicolumn{10}{|c|}{ t2p@EtP5 } \\
\hline C4 - H146 & 0.0041 & 0.0112 & 1.1323 & -0.0020 & 0.0024 & 0.0004 & 0.8220 & 3.135 & 0.64 \\
\hline C7 - H150 & 0.0043 & 0.0113 & 0.1952 & -0.0020 & 0.0024 & 0.0004 & 0.8232 & 3.050 & 0.65 \\
\hline C17 - H138 & 0.0049 & 0.0141 & 0.5209 & -0.0023 & 0.0029 & 0.0006 & 0.7879 & 3.002 & 0.78 \\
\hline C18 - H148 & 0.0033 & 0.0092 & 0.6170 & -0.0015 & 0.0019 & 0.0004 & 0.7825 & 3.258 & 0.51 \\
\hline C28 - H144 & 0.0065 & 0.0183 & 2.6176 & -0.0033 & 0.0040 & 0.0006 & 0.8412 & 2.846 & 1.06 \\
\hline C40 - C145 & 0.0021 & 0.0053 & 2.0299 & -0.0009 & 0.0011 & 0.0002 & 0.8101 & 4.171 & 0.30 \\
\hline C39 - H142 & 0.0059 & 0.0151 & 0.3509 & -0.0027 & 0.0033 & 0.0005 & 0.8430 & 2.884 & 0.88 \\
\hline C51 - H146 & 0.0063 & 0.0167 & 1.5529 & -0.0031 & 0.0036 & 0.0006 & 0.8479 & 2.855 & 0.98 \\
\hline C50 - H141 & 0.0046 & 0.0113 & 0.6777 & -0.0020 & 0.0024 & 0.0004 & 0.8388 & 3.062 & 0.65 \\
\hline C28 - H139 & 0.0035 & 0.0100 & 0.3154 & -0.0016 & 0.0020 & 0.0005 & 0.7791 & 3.185 & 0.55 \\
\hline C27 - H148 & 0.0027 & 0.0077 & 0.2193 & -0.0012 & 0.0016 & 0.0003 & 0.7820 & 3.260 & 0.43 \\
\hline O62 - H142 & 0.0023 & 0.0089 & 0.0512 & -0.0014 & 0.0018 & 0.0004 & 0.7562 & 3.257 & 0.48 \\
\hline O60 - H139 & 0.0067 & 0.0212 & 0.1705 & -0.0044 & 0.0048 & 0.0005 & 0.9017 & 2.689 & 1.30 \\
\hline O61 - H150 & 0.0044 & 0.0151 & 0.5207 & -0.0028 & 0.0033 & 0.0005 & 0.8539 & 2.918 & 0.89 \\
\hline O58 - H138 & 0.0067 & 0.0205 & 0.2481 & -0.0042 & 0.0047 & 0.0004 & 0.9048 & 2.662 & 1.26 \\
\hline H70 - H150 & 0.0050 & 0.0162 & 0.9773 & -0.0027 & 0.0034 & 0.0007 & 0.7956 & 2.526 & 0.90 \\
\hline H95 - H150 & 0.0048 & 0.0124 & 0.1193 & -0.0024 & 0.0028 & 0.0003 & 0.8752 & 2.431 & 0.74 \\
\hline H73 - H139 & 0.0061 & 0.0162 & 0.1626 & -0.0031 & 0.0036 & 0.0005 & 0.8624 & 2.342 & 0.96 \\
\hline H86 - H141 & 0.0042 & 0.0117 & 0.1649 & -0.0022 & 0.0025 & 0.0004 & 0.8563 & 2.500 & 0.69 \\
\hline H92 - H138 & 0.0064 & 0.0204 & 0.6969 & -0.0036 & 0.0043 & 0.0008 & 0.8222 & 2.376 & 1.16 \\
\hline H76 - H148 & 0.0037 & 0.0106 & 0.0478 & -0.0019 & 0.0023 & 0.0004 & 0.8393 & 2.556 & 0.62 \\
\hline H79 - H142 & 0.0053 & 0.0136 & 0.1439 & -0.0027 & 0.0031 & 0.0004 & 0.8838 & 2.397 & 0.82 \\
\hline H82 - H149 & 0.0025 & 0.0072 & 0.0463 & -0.0011 & 0.0015 & 0.0003 & 0.7770 & 2.762 & 0.40 \\
\hline H89 - H149 & 0.0036 & 0.0098 & 0.0562 & -0.0018 & 0.0021 & 0.0003 & 0.8427 & 2.569 & 0.57 \\
\hline
\end{tabular}




\begin{tabular}{|c|c|c|c|c|c|c|c|c|c|}
\hline Interactions & $\rho_{\mathrm{BCP}}$ & $\nabla^{2} \rho_{\mathrm{BCP}}$ & $\epsilon$ & $\mathrm{V}_{\mathrm{BCP}}$ & $\mathrm{G}_{\mathrm{BCP}}$ & $\mathrm{H}_{\mathrm{BCP}}$ & $\left(-\mathrm{G}_{\mathrm{BCP}}\right) / \mathrm{V}_{\mathrm{BCP}}$ & $\mathrm{R}(\AA)$ & $\begin{array}{l}\mathrm{E}_{\mathrm{HB}}(\mathrm{kcal} \\
\left.\mathrm{mol}^{-1}\right)\end{array}$ \\
\hline \multicolumn{10}{|c|}{ c2p@EtP5 } \\
\hline C8 - H142 & 0.0071 & 0.0190 & 1.6814 & -0.0033 & 0.0040 & 0.0007 & 0.8266 & 2.804 & 1.09 \\
\hline C15 - H142 & 0.0067 & 0.0183 & 1.5229 & -0.0032 & 0.0039 & 0.0007 & 0.8271 & 2.882 & 1.05 \\
\hline C18 - H150 & 0.0041 & 0.0107 & 0.2262 & -0.0019 & 0.0023 & 0.0004 & 0.8258 & 3.050 & 0.61 \\
\hline C21 - H141 & 0.0055 & 0.0151 & 1.3981 & -0.0028 & 0.0033 & 0.0005 & 0.8464 & 2.957 & 0.88 \\
\hline C26 - H141 & 0.0041 & 0.0107 & 1.0244 & -0.0020 & 0.0023 & 0.0003 & 0.8573 & 3.114 & 0.63 \\
\hline C29 - H148 & 0.0066 & 0.0202 & 0.9919 & -0.0033 & 0.0042 & 0.0009 & 0.7873 & 2.842 & 1.12 \\
\hline C40 - C145 & 0.0041 & 0.0101 & 0.1782 & -0.0017 & 0.0021 & 0.0004 & 0.8110 & 3.649 & 0.57 \\
\hline C39 - H138 & 0.0058 & 0.0142 & 0.2684 & -0.0026 & 0.0031 & 0.0005 & 0.8532 & 2.863 & 0.83 \\
\hline C30 - H138 & 0.0036 & 0.0113 & 0.6087 & -0.0018 & 0.0023 & 0.0005 & 0.7704 & 3.114 & 0.62 \\
\hline C27 - H150 & 0.0066 & 0.0206 & 0.9488 & -0.0034 & 0.0043 & 0.0009 & 0.7907 & 2.785 & 1.15 \\
\hline C50 - H139 & 0.0026 & 0.0070 & 0.2781 & -0.0011 & 0.0014 & 0.0003 & 0.7871 & 3.426 & 0.39 \\
\hline H95 - C145 & 0.0025 & 0.0063 & 0.3685 & -0.0011 & 0.0013 & 0.0003 & 0.8032 & 3.370 & 0.35 \\
\hline C51 - H144 & 0.0085 & 0.0253 & 3.2492 & -0.0043 & 0.0053 & 0.0010 & 0.8136 & 2.702 & 1.43 \\
\hline O64 - H148 & 0.0088 & 0.0261 & 0.1044 & -0.0055 & 0.0060 & 0.0005 & 0.9173 & 2.533 & 1.62 \\
\hline H73 - H137 & 0.0044 & 0.0123 & 0.2597 & -0.0022 & 0.0026 & 0.0004 & 0.8331 & 2.522 & 0.71 \\
\hline H79 - H138 & 0.0034 & 0.0100 & 0.7881 & -0.0017 & 0.0021 & 0.0004 & 0.8042 & 2.683 & 0.56 \\
\hline H86 - H139 & 0.0039 & 0.0104 & 0.0521 & -0.0020 & 0.0023 & 0.0003 & 0.8621 & 2.505 & 0.62 \\
\hline H92 - H139 & 0.0013 & 0.0045 & 0.3662 & -0.0006 & 0.0008 & 0.0003 & 0.6643 & 3.172 & 0.23 \\
\hline H89 - H146 & 0.0060 & 0.0165 & 0.1075 & -0.0032 & 0.0036 & 0.0005 & 0.8710 & 2.308 & 0.98 \\
\hline H76 - H148 & 0.0072 & 0.0234 & 0.4925 & -0.0039 & 0.0049 & 0.0010 & 0.7992 & 2.352 & 1.31 \\
\hline
\end{tabular}




\begin{tabular}{|c|c|c|c|c|c|c|c|c|c|}
\hline Interactions & $\rho_{\mathrm{BCP}}$ & $\nabla^{2} \rho_{\mathrm{BCP}}$ & $\epsilon$ & $\mathrm{V}_{\mathrm{BCP}}$ & $\mathrm{G}_{\mathrm{BCP}}$ & $\mathrm{H}_{\mathrm{BCP}}$ & $\left(-\mathrm{G}_{\mathrm{BCP}}\right) / \mathrm{V}_{\mathrm{BCP}}$ & $\mathrm{R}(\AA)$ & $\begin{array}{l}\mathrm{E}_{\mathrm{HB}}(\mathrm{kcal} \\
\left.\mathrm{mol}^{-1}\right)\end{array}$ \\
\hline \multicolumn{10}{|c|}{ 1ClBu@EtP5 } \\
\hline C6 - H143 & 0.0060 & 0.0149 & 1.0321 & -0.0028 & 0.0033 & 0.0005 & 0.8531 & 2.888 & 0.88 \\
\hline C18 - H145 & 0.0056 & 0.0146 & 1.2426 & -0.0027 & 0.0032 & 0.0005 & 0.8549 & 2.913 & 0.86 \\
\hline C29 - H146 & 0.0058 & 0.0148 & 1.6202 & -0.0028 & 0.0032 & 0.0005 & 0.8537 & 2.931 & 0.87 \\
\hline C37 - H146 & 0.0042 & 0.0109 & 1.1246 & -0.0020 & 0.0024 & 0.0004 & 0.8457 & 3.113 & 0.63 \\
\hline C39 - H139 & 0.0045 & 0.0118 & 0.1330 & -0.0021 & 0.0025 & 0.0004 & 0.8268 & 3.014 & 0.68 \\
\hline C40 - H147 & 0.0046 & 0.0117 & 0.1787 & -0.0021 & 0.0025 & 0.0004 & 0.8450 & 2.998 & 0.68 \\
\hline C41 - H142 & 0.0040 & 0.0106 & 1.0481 & -0.0019 & 0.0023 & 0.0004 & 0.8265 & 3.106 & 0.61 \\
\hline C50 - H142 & 0.0066 & 0.0165 & 0.6065 & -0.0031 & 0.0036 & 0.0005 & 0.8611 & 2.826 & 0.97 \\
\hline C49 - H147 & 0.0074 & 0.0224 & 0.3692 & -0.0038 & 0.0047 & 0.0009 & 0.8109 & 2.682 & 1.27 \\
\hline C5 - H148 & 0.0068 & 0.0185 & 0.4362 & -0.0032 & 0.0039 & 0.0007 & 0.8200 & 2.718 & 1.05 \\
\hline C19 - H140 & 0.0048 & 0.0148 & 2.4876 & -0.0025 & 0.0031 & 0.0006 & 0.8116 & 2.916 & 0.84 \\
\hline O60 - H140 & 0.0047 & 0.0162 & 3.7881 & -0.0030 & 0.0035 & 0.0005 & 0.8522 & 2.857 & 0.95 \\
\hline O62 - H139 & 0.0056 & 0.0187 & 0.7497 & -0.0036 & 0.0042 & 0.0005 & 0.8758 & 2.777 & 1.12 \\
\hline H89 - Cl149 & 0.0042 & 0.0126 & 0.5354 & -0.0020 & 0.0026 & 0.0005 & 0.7886 & 3.168 & 0.70 \\
\hline H95 - Cl149 & 0.0047 & 0.0131 & 0.0187 & -0.0022 & 0.0027 & 0.0006 & 0.7985 & 3.104 & 0.74 \\
\hline H82 - Cl149 & 0.0066 & 0.0186 & 0.0889 & -0.0031 & 0.0039 & 0.0008 & 0.7948 & 2.935 & 1.04 \\
\hline H76 - Cl149 & 0.0066 & 0.0192 & 0.0976 & -0.0031 & 0.0040 & 0.0008 & 0.7856 & 2.910 & 1.06 \\
\hline H70 - Cl149 & 0.0067 & 0.0193 & 0.0935 & -0.0031 & 0.0040 & 0.0008 & 0.7886 & 2.913 & 1.07 \\
\hline H79 - H139 & 0.0041 & 0.0118 & 0.5799 & -0.0020 & 0.0025 & 0.0005 & 0.8178 & 2.591 & 0.67 \\
\hline H89 - H147 & 0.0045 & 0.0153 & 0.9245 & -0.0026 & 0.0032 & 0.0006 & 0.8108 & 2.566 & 0.86 \\
\hline H92 - H138 & 0.0014 & 0.0048 & 0.2643 & -0.0006 & 0.0009 & 0.0003 & 0.6708 & 3.093 & 0.24 \\
\hline H86 - H138 & 0.0016 & 0.0052 & 0.2165 & -0.0007 & 0.0010 & 0.0003 & 0.6948 & 2.993 & 0.27 \\
\hline
\end{tabular}




\begin{tabular}{|c|c|c|c|c|c|c|c|c|c|}
\hline Interactions & $\rho_{\mathrm{BCP}}$ & $\nabla^{2} \rho_{\mathrm{BCP}}$ & $\epsilon$ & $\mathrm{V}_{\mathrm{BCP}}$ & $\mathrm{G}_{\mathrm{BCP}}$ & $\mathrm{H}_{\mathrm{BCP}}$ & $\left(-\mathrm{G}_{\mathrm{BCP}}\right) / \mathrm{V}_{\mathrm{BCP}}$ & $\mathrm{R}(\AA)$ & $\begin{array}{l}\mathrm{E}_{\mathrm{HB}}(\mathrm{kcal} \\
\left.\mathrm{mol}^{-1}\right)\end{array}$ \\
\hline \multicolumn{10}{|c|}{ 2ClBu@EtP5 } \\
\hline C5 - H143 & 0.0076 & 0.0208 & 0.5416 & -0.0037 & 0.0044 & 0.0008 & 0.8262 & 3.576 & 1.19 \\
\hline C19 - H140 & 0.0054 & 0.0162 & 10.0587 & -0.0028 & 0.0034 & 0.0006 & 0.8201 & 2.916 & 0.92 \\
\hline C29 - H145 & 0.0036 & 0.0095 & 0.2320 & -0.0016 & 0.0020 & 0.0004 & 0.8167 & 3.907 & 0.54 \\
\hline C30 - H139 & 0.0066 & 0.0186 & 3.8935 & -0.0033 & 0.0040 & 0.0007 & 0.8282 & 2.882 & 1.07 \\
\hline C40 - H142 & 0.0049 & 0.0127 & 1.7488 & -0.0024 & 0.0028 & 0.0004 & 0.8519 & 3.784 & 0.74 \\
\hline C48 - H142 & 0.0054 & 0.0144 & 1.4957 & -0.0027 & 0.0031 & 0.0005 & 0.8525 & 3.049 & 0.84 \\
\hline C51 - H143 & 0.0042 & 0.0117 & 1.6131 & -0.0020 & 0.0025 & 0.0005 & 0.8075 & 4.114 & 0.66 \\
\hline $061-\mathrm{Cl} 149$ & 0.0053 & 0.0215 & 2.9219 & -0.0035 & 0.0044 & 0.0009 & 0.7899 & 4.254 & 1.20 \\
\hline C18 - Cl149 & 0.0054 & 0.0169 & 1.9002 & -0.0024 & 0.0033 & 0.0009 & 0.7291 & 4.449 & 0.89 \\
\hline O64 - H145 & 0.0058 & 0.0184 & 0.2913 & -0.0037 & 0.0041 & 0.0005 & 0.8870 & 5.177 & 1.11 \\
\hline O57 - H146 & 0.0111 & 0.0342 & 0.0307 & -0.0069 & 0.0077 & 0.0008 & 0.8964 & 5.068 & 2.08 \\
\hline O59 - H147 & 0.0048 & 0.0154 & 0.8320 & -0.0029 & 0.0034 & 0.0005 & 0.8655 & 4.253 & 0.92 \\
\hline C7 - Cl149 & 0.0029 & 0.0084 & 0.8087 & -0.0012 & 0.0016 & 0.0005 & 0.7221 & 4.622 & 0.44 \\
\hline H76 - Cl149 & 0.0074 & 0.0226 & 0.0778 & -0.0036 & 0.0046 & 0.0010 & 0.7848 & 2.910 & 1.25 \\
\hline H70 - Cl149 & 0.0086 & 0.0306 & 0.0680 & -0.0050 & 0.0063 & 0.0013 & 0.7927 & 2.913 & 1.71 \\
\hline H95 - Cl149 & 0.0061 & 0.0167 & 0.0615 & -0.0028 & 0.0035 & 0.0007 & 0.8005 & 3.104 & 0.93 \\
\hline H82 - H146 & 0.0070 & 0.0219 & 1.0554 & -0.0037 & 0.0046 & 0.0009 & 0.8039 & 3.789 & 1.23 \\
\hline H95 - H147 & 0.0049 & 0.0167 & 0.9250 & -0.0028 & 0.0035 & 0.0007 & 0.7993 & 3.943 & 0.93 \\
\hline H44 - H146 & 0.0058 & 0.0169 & 0.3164 & -0.0028 & 0.0035 & 0.0007 & 0.8044 & 4.165 & 0.95 \\
\hline H100 - H147 & 0.0053 & 0.0152 & 0.2470 & -0.0027 & 0.0033 & 0.0005 & 0.8402 & 4.445 & 0.88 \\
\hline $061-\mathrm{Cl} 149$ & 0.0053 & 0.0215 & 2.9219 & -0.0035 & 0.0045 & 0.0009 & 0.7778 & 3.439 & 1.21 \\
\hline
\end{tabular}


Table S5 Summary of interactions from QTAIM analysis.

\begin{tabular}{cccccc}
\hline Complex & C-H--- $\pi$ & O---H & H---H & Cl---H & Cl---O \\
\hline EtP5-1P & 11 & 2 & 10 & --- & --- \\
EtP5-t2p & 10 & 4 & 9 & --- & --- \\
EtP5-c2p & 13 & 1 & 6 & --- & --- \\
& & & & & \\
EtP5-1ClBu & 11 & 2 & 4 & 5 & --- \\
EtP5-2ClBu & 9 & 3 & 4 & 1 & 1 \\
& & & & & \\
\hline
\end{tabular}



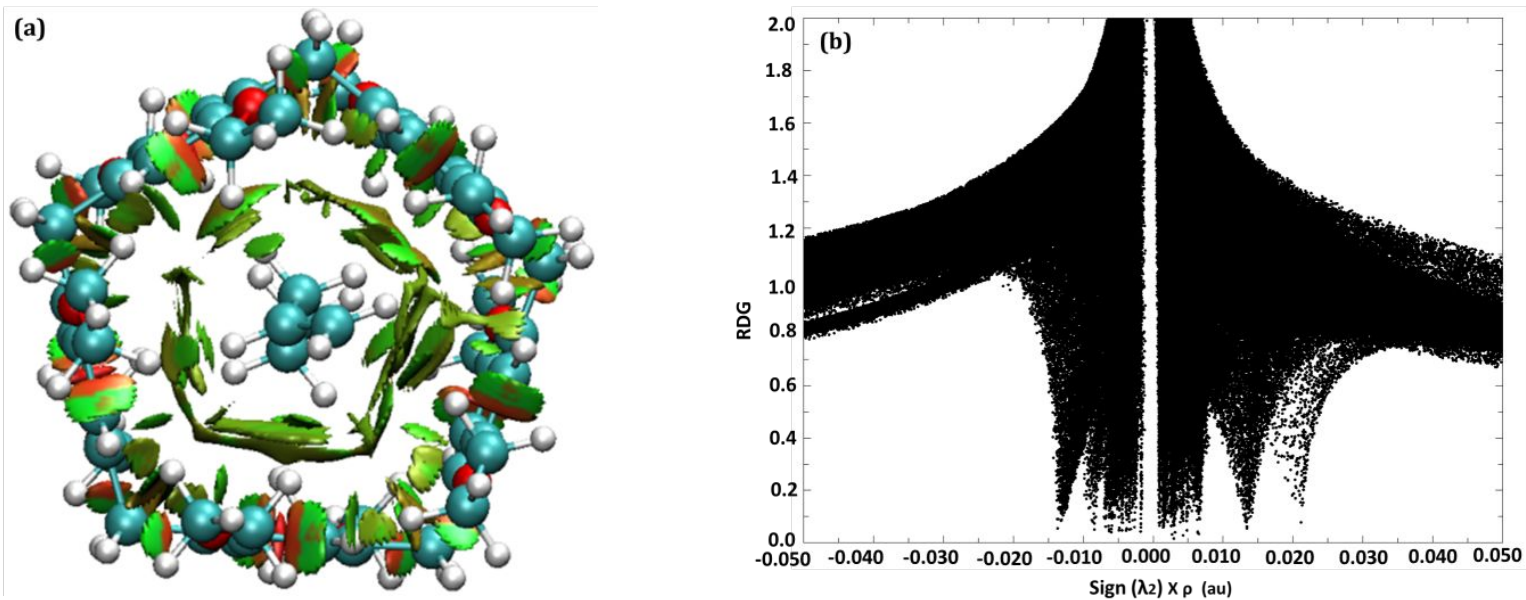

1-P@EtP5
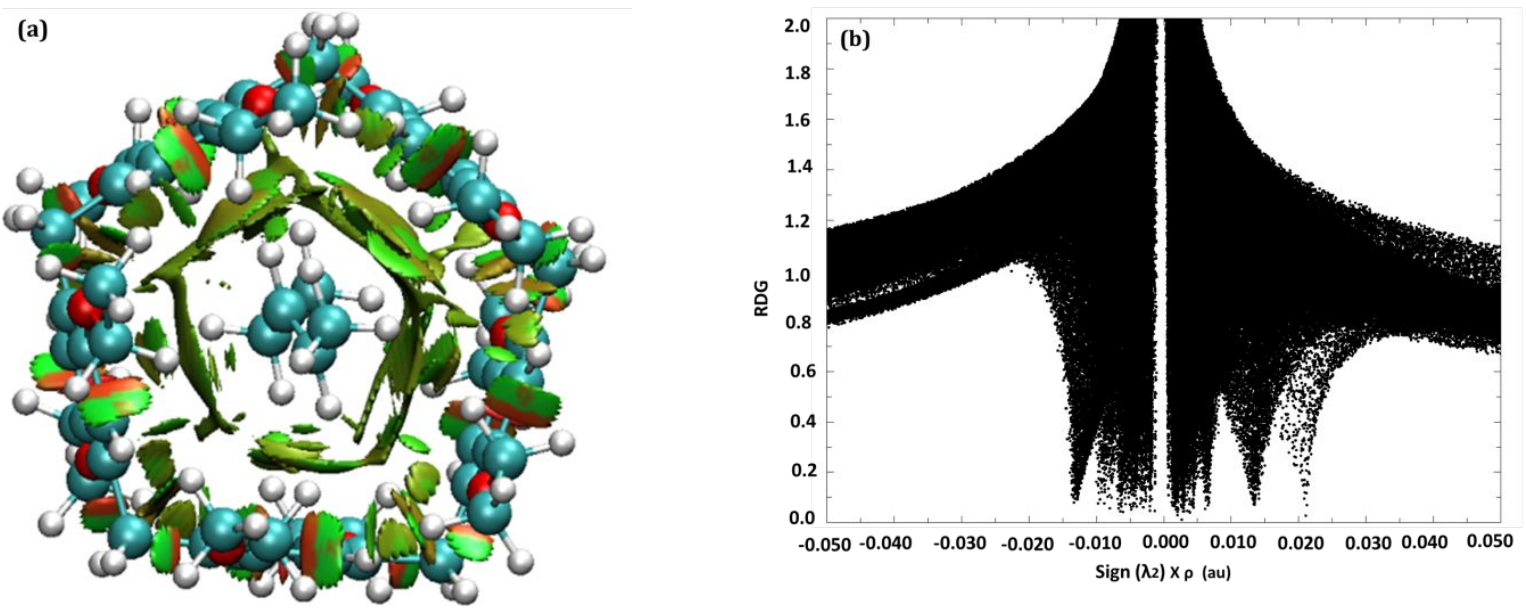

t2p@EtP5
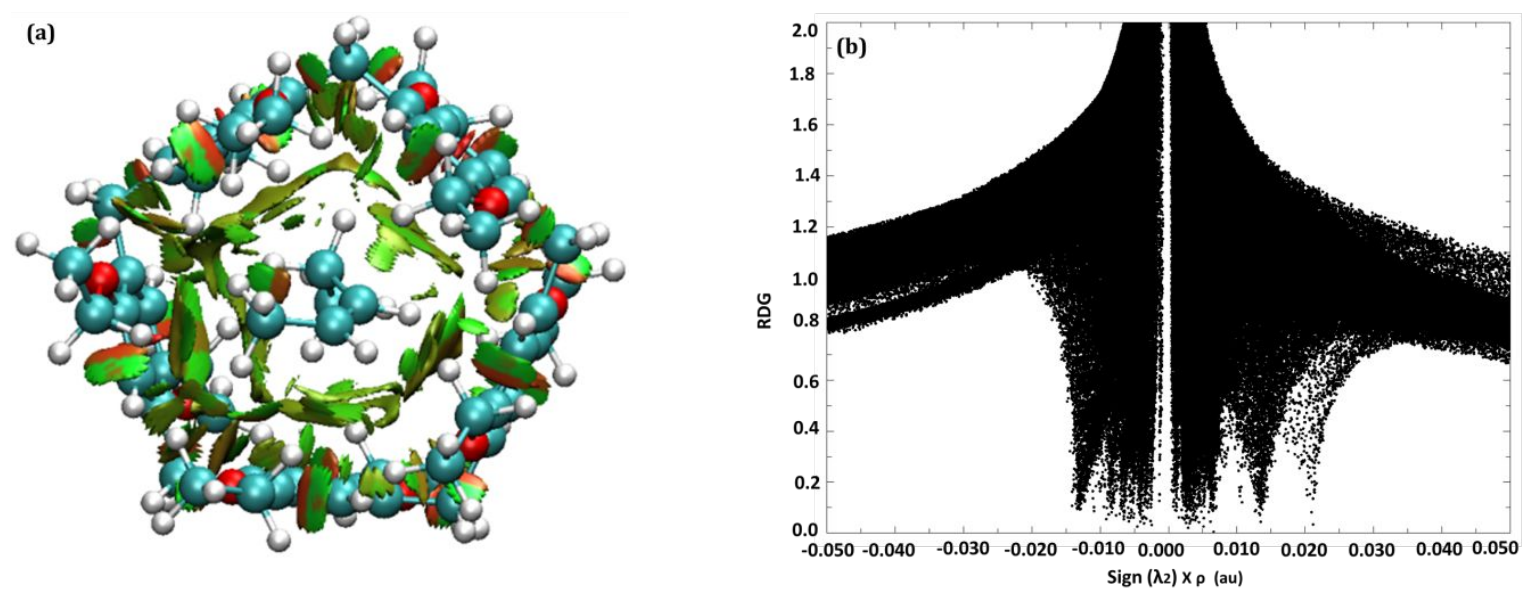

c2p@EtP5

Continued... 

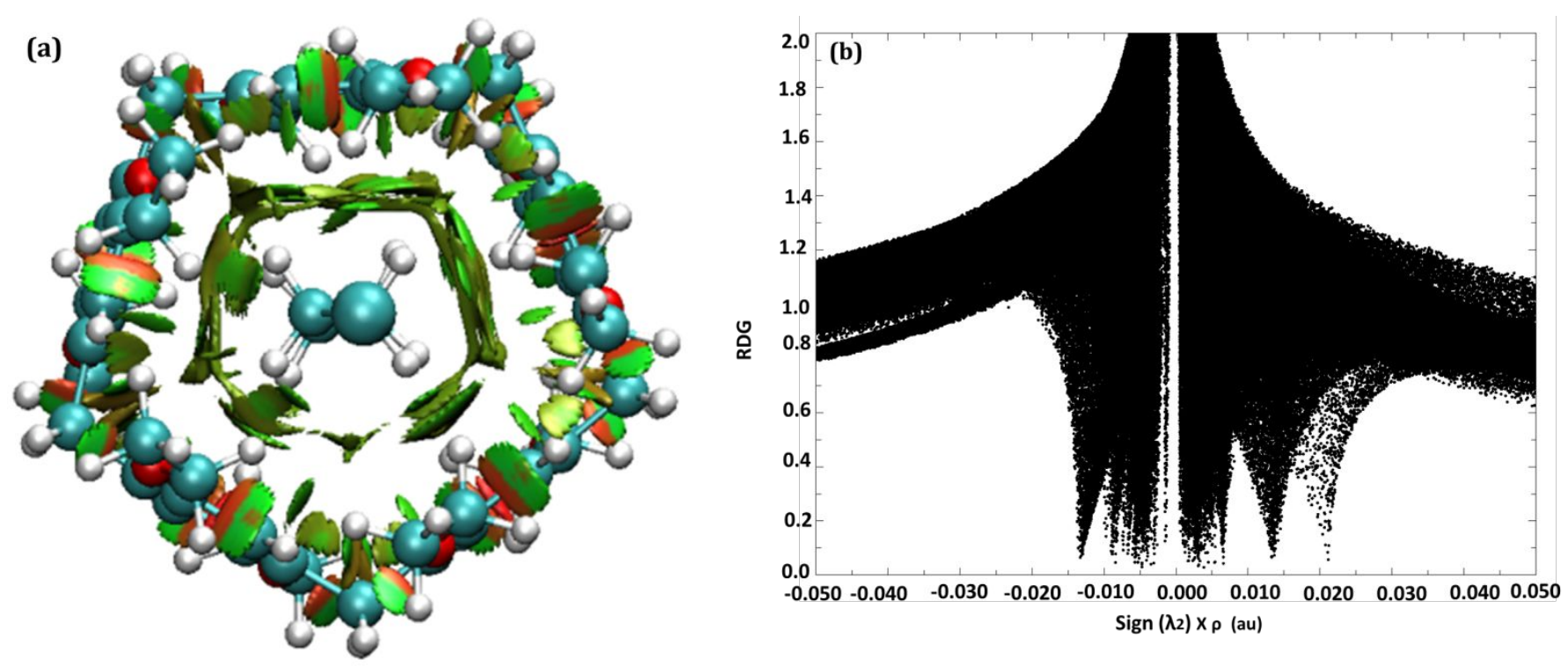

\section{ClBu@EtP5}
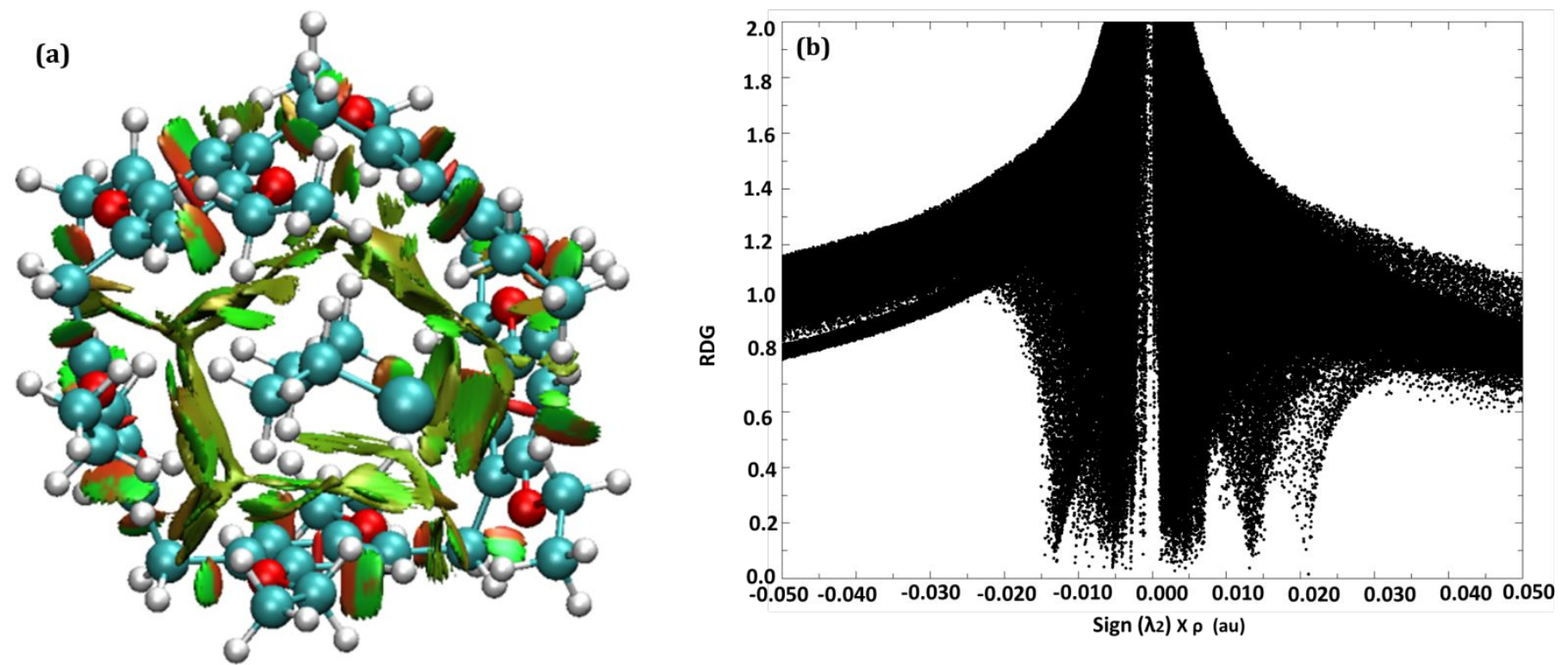

\section{ClBu@EtP5}

Fig. S6 (a) Color-filled RDG isosurface plot: Non-covalent interaction (NCI) regions in EtP5 bound complexes with sign $\left(\lambda_{2}\right)$ range -0.050 to +0.050 au (green colored isosurface denotes weak H-bonds and the red colored isosurface stands for steric effects). (b) NCI index plot: The plot of the reduced density gradient (RDG) on the Yaxis vs. ( $\operatorname{sign}(\lambda 2) \rho$ values) on the $\mathrm{X}$-axis. 

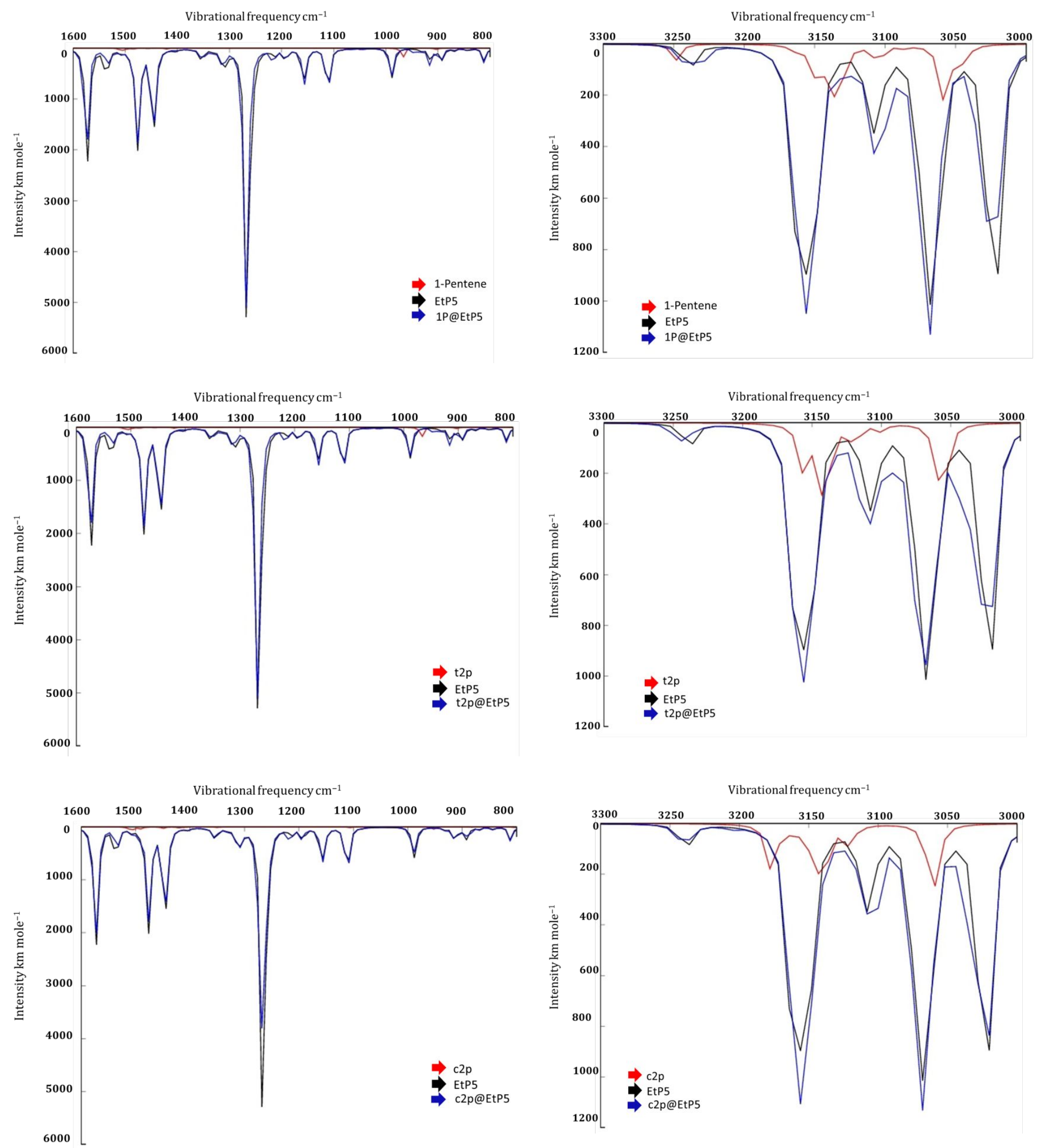

Continued... 

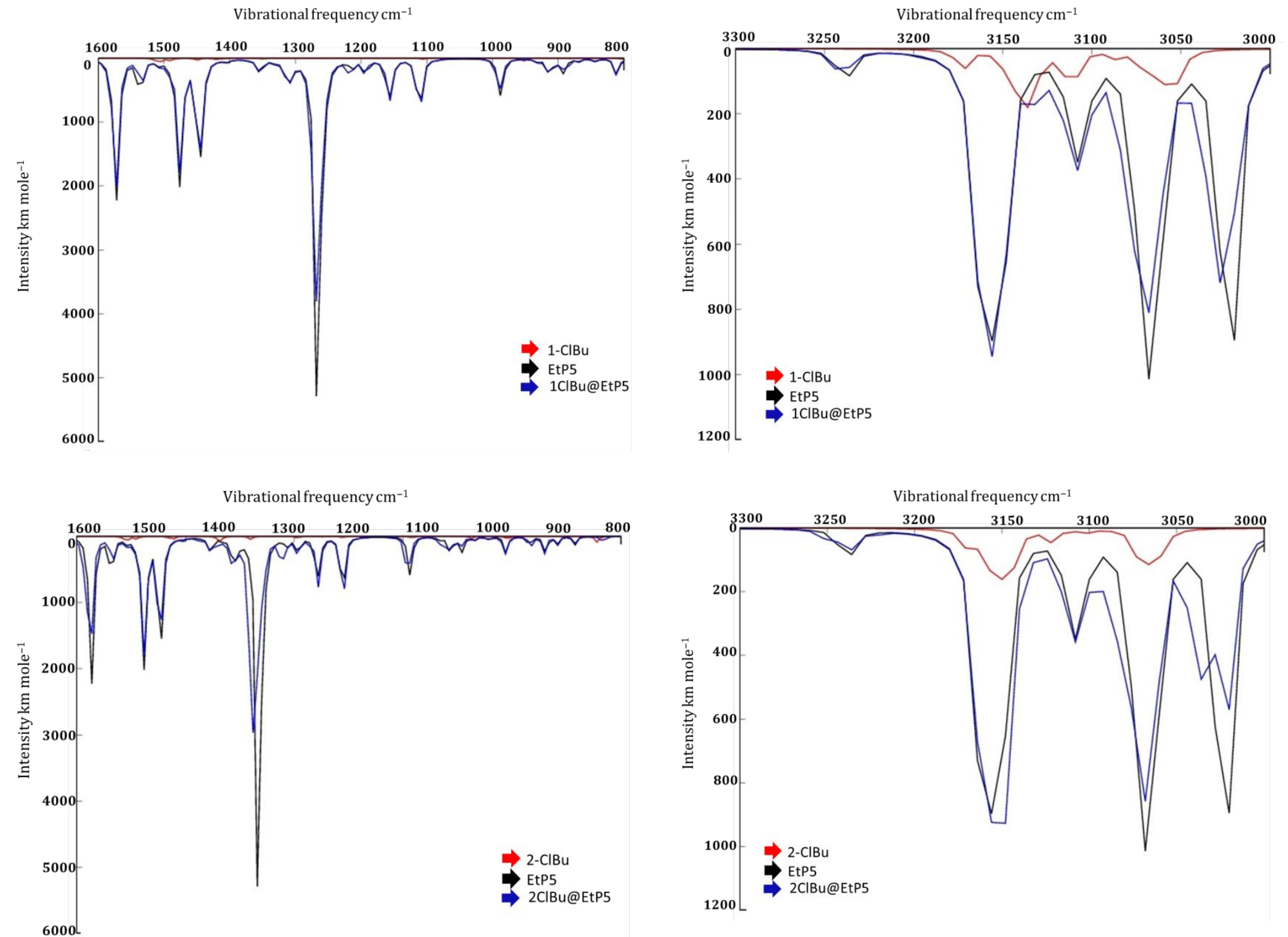

Fig. S7 IR spectra for pentene and chlorobutane complexes of EtP5 